\title{
Hepatoprotective Effect of Allium hooshidaryae Hydroalcoholic Extract on Acetaminophen-Induced Hepatotoxicity in Male Rats
}

\author{
Saeed Ovji ${ }^{1,2}$, Dara Dastan ${ }^{1,3}$, Sara Soleimani Asl ${ }^{4}$ and Amir Nili-Ahmadabadi ${ }^{1,2,{ }^{*}}$ \\ ${ }^{1}$ Medicinal Plants and Natural Products Research Center, Hamadan University of Medical Sciences, Hamadan, Iran \\ ${ }^{2}$ Department of Pharmacology and Toxicology, School of Pharmacy, Hamadan University of Medical Sciences, Hamadan, Iran \\ ${ }^{3}$ Department of Pharmacognosy and Pharmaceutical Biotechnology, School of Pharmacy, Hamadan University of Medical Sciences, Hamadan, Iran \\ ${ }^{4}$ Anatomy Department, School of Medicine, Hamadan University of Medical Sciences, Hamadan, Iran \\ "Corresponding author: Medicinal Plants and Natural Products Research Center, Hamadan University of Medical Sciences, Hamadan, Iran Email: amirnili54@gmail.com
}

Received 2019 December 03; Revised 2020 April 15; Accepted 2020 June 26.

\section{Abstract}

Background: Acetaminophen (APAP) hepatotoxicity is a highly prevalent drug used for the treatment of pain. Allium hooshidaryae is widely used as herbal medicine in western Iran.

Objectives: In this study, we investigated the protective effects of A. hooshidaryae methanolic extract (AhME) on liver damage induced by APAP.

Methods: Rats were divided into six groups. Groups 1 (negative control) and 2 (positive control) received normal saline; groups 3 received $200 \mathrm{mg} / \mathrm{kg}$ of AhME, and groups $4-6$ received AhME at the doses of 50,100, and $200 \mathrm{mg} / \mathrm{kg}$ once daily, by gavage, respectively. After the 14 days, except for groups 1 and 3, the other groups received $2 \mathrm{~g} / \mathrm{kg}$ of APAP. After 48 hours, hepatic tissue and blood sample were taken for histopathology and biochemical experiments.

Results: APAP induced a notable increase in serum levels of aspartate transaminase (AST, $\mathrm{P}<0.001$ ), alanine transaminase (ALT, $\mathrm{P}$ $<0.001$ ), alkaline phosphatase (ALP, $\mathrm{P}<0.001$ ), lactate dehydrogenase (LDH, $\mathrm{P}<0.01$ ), nitric oxide (NO, $\mathrm{P}<0.001$ ), and lipid peroxidation (LPO, $\mathrm{P}<0.001$ ) levels in hepatic tissue. It also significantly reduced the level of total antioxidant capacity (TAC), total thiol groups (TTGs) as well as glutathione contents (GSH) in hepatic tissue (For all $\mathrm{P}<0.001$ ). Following the use of AhME, a noteworthy improvement was observed in hepatic serum enzymes and oxidant/antioxidant parameters in liver tissue.

Conclusions: This study demonstrated that AhME prevents APAP-induced hepatotoxicity through improvement in the hepatic antioxidant status.

Keywords: Allium hooshidaryae, Oxidative Stress, Acetaminophen, Hepatotoxicity

\section{Background}

Acetaminophen and/or N-acetyl-p-aminophenol (APAP) is commonly administered as an analgesic drug worldwide. At remedial doses, APAP is predominantly eliminated by phase 2 enzymes to inert conjugates. A small amount of this medicine being metabolized by cytochrome P450 (CYP2E1) to the extremely reactive agents, $\mathrm{N}$-acetyl-p-benzoquinoneimine (NAPQI). NAPQI is eliminated by combining with glutathione $(\mathrm{GSH})$ to create an inert conjugate. The saturated phase-2 pathways can lead to high NAPQI at APAP overdose, GSH depletion, and hepatic dysfunction $(1,2)$.

In recent years, developing countries provide their health needs with herbal medicine (3). Belonging to the Amaryllidaceae family, the Allium genus has about 700 different species, such as Allium cepa (onion), A. schoenoprasum (chive), A. sativum (garlic), A. tuberosum (garlic chive).
Their antioxidant potential is due to the presence of thiosulfinates and other organosulfur, saponins, flavonoids, and phenolic compounds, which are nutritionally and economically valuable (4).

According to recent meta-analyses studies, these species have beneficial effects on blood pressure, lipid profiles, and other cardiovascular risk factors (5-8).

\section{Objectives}

The A. hooshidaryae is a native plant in the Kurdistan Region, consumed by local people as an herbal medicine for various diseases. Given the limited evidence regarding the therapeutic effects of A. hooshidaryae, the present study aimed to investigate the possible protective effects of methanolic extract of this plant on the hepatotoxicity induced by APAP. 


\section{Methods}

\subsection{Chemicals}

N-acetyl-p-aminophenol (APAP, CAS: 103-90-2), 2thiobarbituric acid (TBA), 5,5'-dithiobis (2-nitrobenzoic acid) (DTNB), N-(1-naphthyl) ethylenediamine dihydrochloride (NED), sulfanilamide, and 2,4,6-Tris(2-pyridyl)s-triazine (TPTZ) were bought from Sigma-Aldrich Chemical Company (St. Louis, MO, USA).

\subsection{Plant Material and Extraction}

The A. hooshidaryae was collected from Iran, Prov. Kordestan, (Hawraman Mountains), and identified by herbarium unit, Natural Products Research Center (NPRC), Hamadan, Iran (NO:274). The aerial parts of A. hooshidaryae were dried in the shade and soaked in equal proportions of water and methanol after crushing for three days. The extract was concentrated by a rotary evaporator at $45^{\circ} \mathrm{C}$ and then fractioned by column chromatography by n-hexane, chloroform, ethyl acetate, and methanol fractions. For this purpose, a silica gel column (30 - 70 mesh) was used as a solid phase. The quality of fractions was evaluated by the ferric reducing/antioxidant power (FRAP) method.

\subsection{Animals}

The animal study protocol was approved by the HUMS Ethics Committee (identification code: IR.UMSHA.REC.1396.365). Male Wistar rats $(250 \pm 20$ gram) were purchased from the animal center of HUMS and kept for one week under suitable conditions (temperatures $22^{\circ} \mathrm{C}-25^{\circ} \mathrm{C}, 12 \mathrm{~h}$ light/dark cycle) and fed with a commercial laboratory diet and water ad libitum.

\subsection{Preliminary Studies}

The preliminary studies revealed that the $A$. hooshidaryae methanolic extract (AhME) has the highest antioxidant potential and was safe in the dose range of $50-200 \mathrm{mg} / \mathrm{kg}$.

\subsection{Study Design}

Thirty-six rats were divided into six groups of six each. Groups 1 (negative control) and 2 (positive control) received normal saline; groups 3 received $200 \mathrm{mg} / \mathrm{kg}$ of AhME, and groups 4 - 6 received AhME at the doses of 50, 100 , and $200 \mathrm{mg} / \mathrm{kg}$ once daily, by gavage, respectively. After the 14 days, except for groups 1 and 3, the other groups received $2 \mathrm{~g} / \mathrm{kg}$ of APAP. After 48 hours, the rats were anesthetized using ether and killed. The blood sample was collected from the rat's heart and centrifuged (3000 rpm, 10 min). The serum sample was removed and kept at $-20^{\circ} \mathrm{C}$. In addition to liver tissue sampling for histological studies, a small portion of hepatic tissue $(100 \mathrm{mg})$ was homogenized in $1 \mathrm{~mL}$ phosphate-buffered saline ( $\mathrm{PBS}, \mathrm{pH}=7.4$ ). The liver homogenate was centrifuged ( $4000 \mathrm{rpm} / 4^{\circ} \mathrm{C} / 5 \mathrm{~min}$ ), and its supernatant was separated and kept at $-80^{\circ} \mathrm{C}$ until the biochemical evaluation.

\subsection{Serum Enzymes Analysis}

The serum alanine transaminase (ALT), lactate dehydrogenase (LDH), aspartate transaminase (AST), and alkaline phosphatase (ALP) values were measured kinetically using commercial kits (Pars Azmoon, Iran).

\subsection{Assessment of Hepatic Oxidative Injury}

In the hepatic tissue, nitric oxide (NO) was detected using Griess reagent as described by Nili-Ahmadabadi et al. (9). Also, total antioxidant capacity (TAC) and lipid peroxidation (LPO) were measured by FRAP and thiobarbituric acid reactive substances (TBARS) methods, as described in the previous study $(9,10)$. Total thiol groups (TTGs) were determined via DTNB as the reagent (11). The GSH hepatic level was assayed based on the kit protocol from ZellBio $\mathrm{GmbH}$ Company, Germany. Finally, protein content was assayed in tissue homogenate supernatant via the Bradford method.

\subsection{Histopathological Consideration}

A piece of liver tissue was held in $10 \%$ neutral buffered formalin for at least $24 \mathrm{~h}$ to be fixed. The paraffinembedded block was prepared and cut into four $\mu$ m thick sections by a microtome instrument. After staining with hematoxylin and eosin (H \& E) dye, stained samples were evaluated under the light microscope (Olympus CX31 microscope) (12).

\subsection{Statistical Analysis}

The data is described as the mean \pm SEM. Differences were determined at 95\% confidence (P-value < 0.05) via analysis of variance (ANOVA) and Tukey's posthoc test. The statistical analysis was conducted using SPSS version 20 (IBM, Armonk, NY, USA).

\section{Results}

\subsection{The Effects of AhME on Serum Liver Enzymes}

The findings showed no sign of toxicity up to 200 $\mathrm{mg} / \mathrm{kg}$ of AhME. As shown in Figure 1, the APAP notable increased AST $(\mathrm{P}<0.001)$, ALT $(\mathrm{P}<0.001), \mathrm{LDH}(\mathrm{P}<0.01)$, and ALP $(\mathrm{P}<0.001)$ serum activity when compared to control group. The AhME decreased the serum levels of hepatic enzymes (AST, ALT, and ALP) in rats exposed to APAP. There was no significant change in serum LDH activity in the pretreatment groups compared to the APAP group. 
A

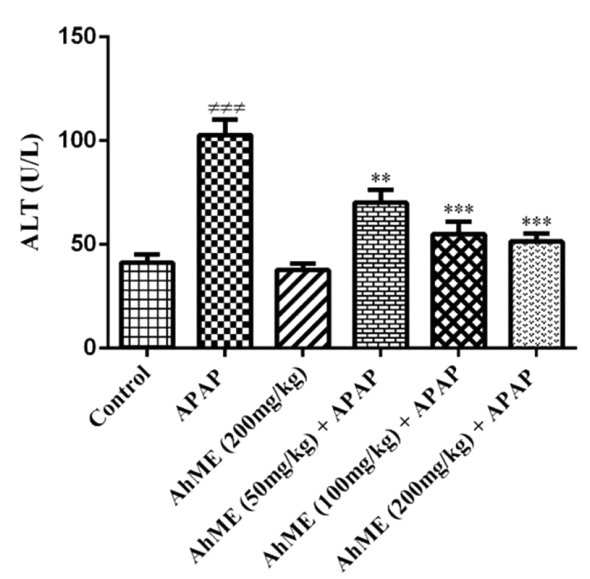

C

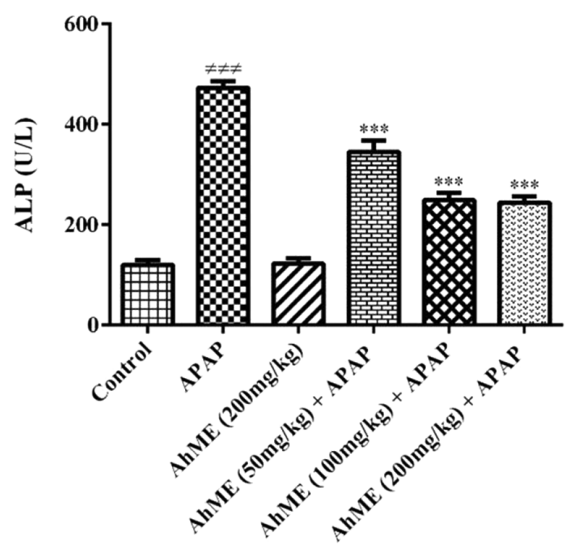

B

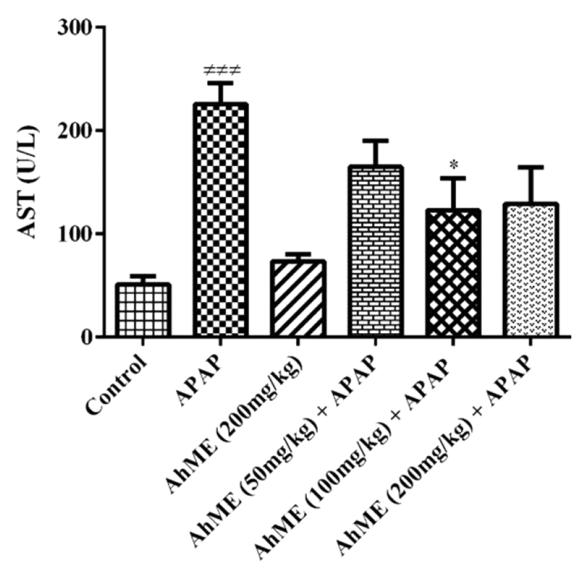

D

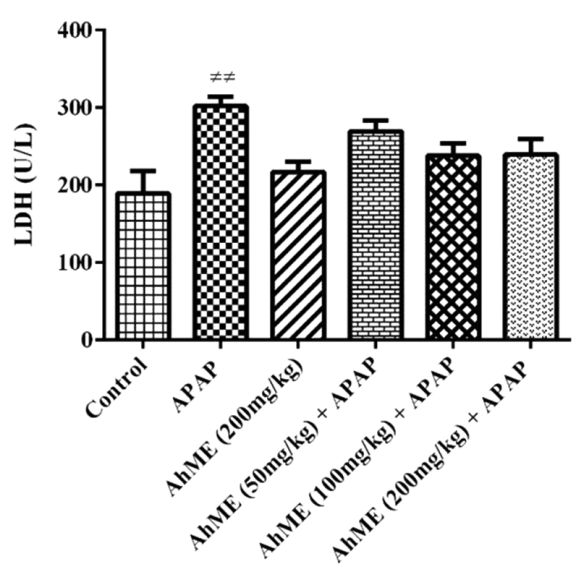

Figure 1. Effects of Allium hooshidaryae methanolic extract (AhME) on serum enzymes of acetaminophen (APAP)-exposed Wistar rat. Statistical analysis used one-way ANOVA with Tukey's test. Values are expressed as means \pm SEM, $\mathrm{n}=6$ for each group. \#\#\#, P<0.001 vs control group; ${ }^{*}, \mathrm{P}<0.05,{ }^{* *}, \mathrm{P}<0.01$ and ${ }^{* * *}, \mathrm{P}<0.001$ vs APAP group. ALT, alanine aminotransferase (A); AST, aspartate aminotransferase (B); ALP, alkaline phosphatase (C); LDH, lactate dehydrogenase (D).

\subsection{The Effects of AhME on Oxidative Stress Markers}

After APAP administration, the levels of LPO and NO were increased (Figure 2, P $<0.001$ ). Also, GSH, TAC, and TTGs levels were decreased in hepatic tissue compared to the control group (Figure $3 ; \mathrm{P}<0.001$ ). Following pretreatment with the AhME, a significant improvement was observed in hepatic TTGs, especially at doses of 100 and 200 $\mathrm{mg} / \mathrm{kg}$ compared to the APAP group $(\mathrm{P}<0.001$ and $\mathrm{P}<$ 0.01, respectively). Besides, administration of AhME can improve the GSH level at the dose of $50 \mathrm{mg} / \mathrm{kg}(\mathrm{P}<0.05)$ and NO and LPO levels (for all doses, $\mathrm{P}<0.001$ ) in hepatic tissue compared to the APAP group.

\subsection{Histopathological Alterations}

We observed the vascular congestion, mononuclear cell infiltration, necrosis, dilation of sinusoids, and increasing kupffer cells in the liver of APAP-treated rats. Following pretreatment with different doses of AhME, a noteworthy improvement was found in some of the pathological alterations like necrosis and vascular congestion (Figure 4).

\section{Discussion}

Our results showed a notable increase in the serum levels of ALT, AST, and LDH in the APAP-treated rats. These results could be due to increased membrane degradation, 
A

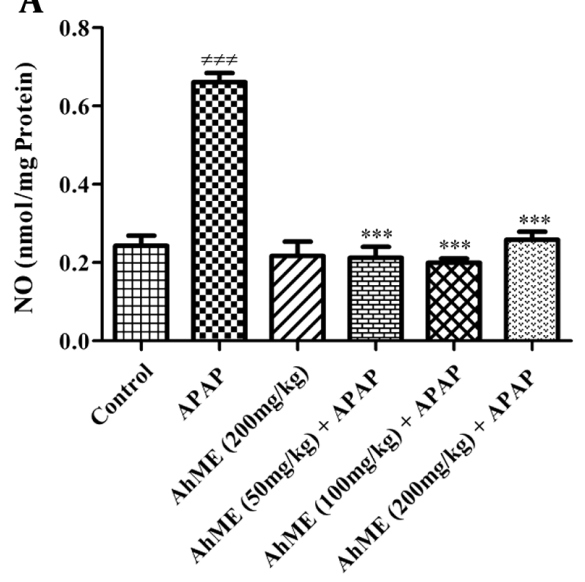

\section{B}

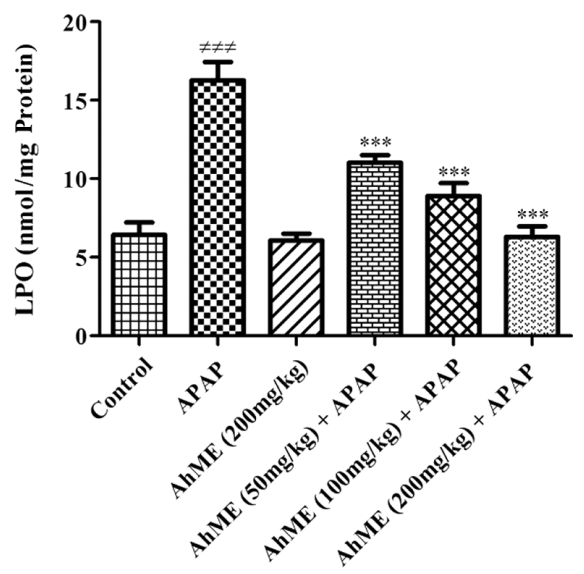

Figure 2. Effects of Allium hooshidaryae methanolic extract (AhME) on oxidant biomarkers of acetaminophen (APAP)-exposed Wistar rat. Statistical analysis used one-way ANOVA with Tukey's test. Values are expressed as means \pm SEM, $n=6$ for each group. \#\#\#, P< 0.001 vs control group; ${ }^{* * *}, \mathrm{P}<0.001$ vs APAP group; NO, nitric oxide (A); LPO, lipid peroxidation (B).

leading to enzyme leakage into circulation(13). Also, the increase in serum level of ALP may be due to the biliary tract impairment and cholestasis (14). Pretreatment with AhME could significantly decrease the serum level of hepatic enzymes, which might be due to its membrane stabilizing activity $(13,15,16)$.

Since the APAP hepatotoxicity is related to oxidative pathways, hepatic oxidant/antioxidant biomarkers were evaluated. Results showed that TAC, TTGs, and GSH decreased in the hepatic tissue of the APAP group, indicating APAP-induced oxidative damage. These findings were in agreement with the other reports (17-21) related to the altered natural metabolism of the APAP and the disruption of the active thiol groups (22). Overall, APAP is metabolized by the cytochrome $\mathrm{P} 450$ enzymes into a reactive metabolite called NAPQI, which reduces the levels of thiol groups and subsequent hepatocellular antioxidant defense $(23,24)$. GSH is part of the natural antioxidant system that counteracts the oxidative stress caused by free radicals (25). GSH is converted to GSSG (glutathione disulfide) by free radicals, causing apoptosis by excessive oxidation of glutathione (26). Also, APAP-induced hepatotoxicity may be associated with the inhibition of useful enzymes in GSH biosynthesis. Singh et al. (27) found that APAP could inhibit the recovery of oxidized GSH to reduced GSH by inhibiting the GSH reductase enzyme and lead to the depletion of thiol reserves. These changes led to the degradation of macromolecules, such as proteins and nucleic acids, and caused the loss of membrane integrity (28).

Following the administration of AhME, a significant improvement was observed in the level of thiol groups, es- pecially GSH. It seems that Allium species can effectively improve the thiol reserves due to having sulfur compounds. For example, the allicin derivative products (diallyl disulfide, diallyl trisulfide) in garlic essential oils have shown suitable antioxidant activities. Also, the onion has antioxidant potential due to dipropyl disulfide and dipropyl trisulfide (29).

LPO is the degradation of lipid caused by the removal of electrons from the cell membrane by free radicals (30, 31). APAP poisoning can cause the generation of reactive oxygen species (ROS) to leak out of the mitochondria, resulting in LPO. The AhME caused a significant decrease in LPO level due to its membrane-stabilizing properties. In the previous studies, the membrane stabilizing properties of some Allium species have been studied by Sharma et al. (32) and Anitha et al. (33).

NO and its derivatives play crucial roles in liver physiology and pathophysiology. It is mainly made by the endothelial nitric oxide synthase (eNOS) and usually has a protective role against diseases. However, excessive production of NO can result in the overproduction of reactive nitrogen species (RNS) after reaction with superoxide anions and the production of active peroxynitrite species, leading to oxidative damage (34-36). In the present study, APAP increased hepatic NO and significantly reduced this chemical mediator in contrast with AhME. Elevated hepatic NO levels following exposure to APAP may be associated with the induction of nitric oxide (iNOS) activity by this drug (37). It seems that active substances in Allium species such as s-allyl cysteine (SAC) and diallyl sulfide prevent the increase of peroxynitrite level and the occurrence 
A

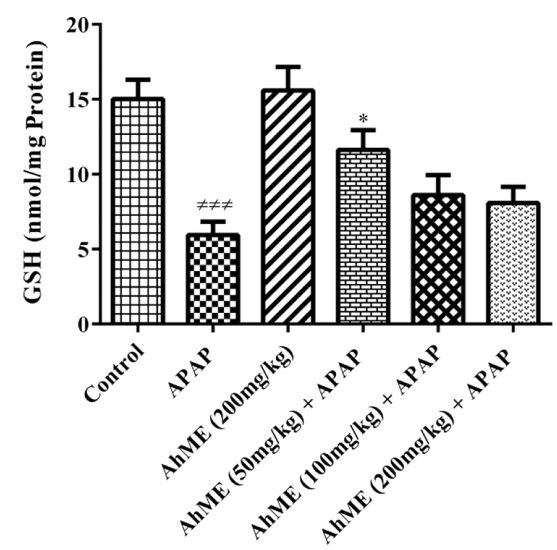

B

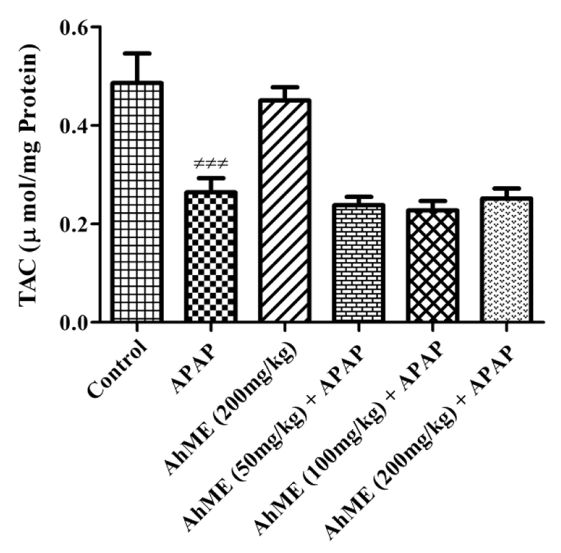

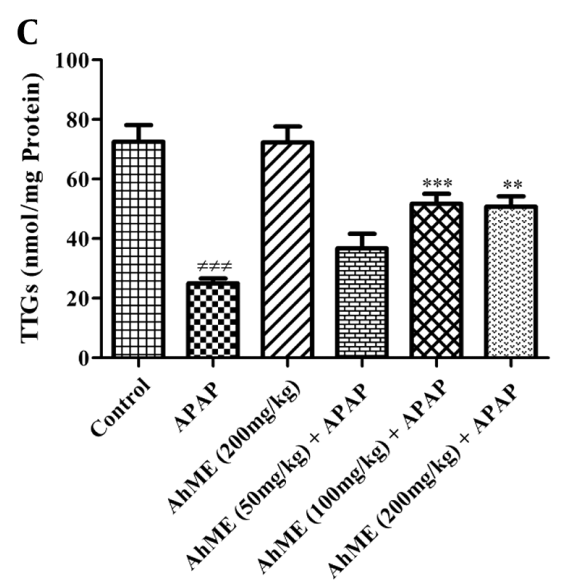

Figure 3. Effects of Allium hooshidaryae methanolic extract (AhME) on antioxidative biomarkers of acetaminophen (APAP)-exposed Wistar rat. Statistical analysis used one-way ANOVA with Tukey's test. Values are expressed as means \pm SEM, $\mathrm{n}=6$ for each group. \#\#\#, $\mathrm{P}<0.001$ vs control group; ${ }^{*}, \mathrm{P}<0.05,{ }^{* *}, \mathrm{P}<0.01$ and ${ }^{* * *}, \mathrm{P}<0.001$ vs APAP group. GSH, glutathione (A); TAC, total antioxidant capacity (B); TTG, total thiol group (C).

of nitrosative stress by reducing the activity of this enzyme and preventing inflammatory reactions in liver tissue (38, 39).

In conclusion, this data suggests that A. hooshidaryae may prevent APAP hepatotoxicity by improving the hepatic thiol reserves. Therefore, this herbal medicine may be used as a complementary treatment for other liver diseases.

\section{Footnotes}

Authors' Contribution: Saeed Ovji helped performed the experimental parts and drafted the manuscript as his Pharm. Dara Dastan was the co-supervisor to the thesis. Sara Soleimani Asl was the advisers of the study. Amir Nili-Ahmadabadi conceived and supervised the study and edited the manuscript.
Conflict of Interests: No conflict of interest declared.

Ethical Approval: The ethical approval code was IR.UMSHA.REC.1396.365.

Funding/Support: This work was financially supported (grant no.: 9605173208) by the Vice-Chancellor of Research and Technology, Hamadan University of Medical Sciences, Hamadan, I.R. Iran. 

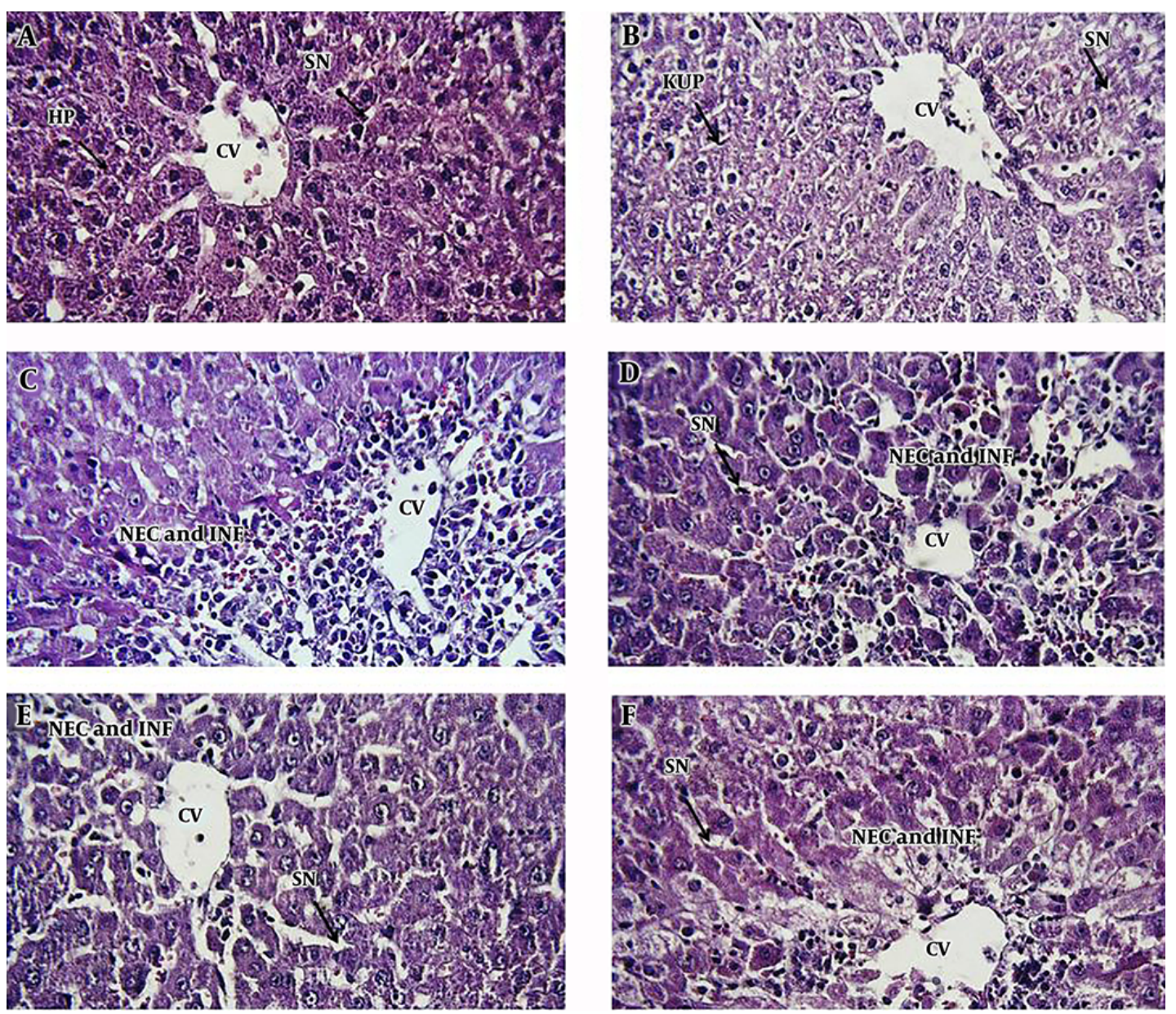

Figure 4. Photomicrographs of rat liver tissue in different groups: A, negative control group; B, AhME control group; C, acetaminophen (APAP; 2 g/kg); D, AhME (50 mg/kg) + APAP (2 g/kg); E, AhME (100 mg/kg) + APAP (2 g/kg); F, AhME (200 mg/kg) + APAP (2 g/kg). Original magnification of all images is $40 \times$. CV, central vein; HP, hepatocyte; SN, sinusoid; KUP, kupffer cells; NEC, necrosis; INF, infiltration.

\section{References}

1. Ramachandran A, Jaeschke H. Mechanisms of acetaminophen hepatotoxicity and their translation to the human pathophysiology. J Clin Transl Res. 2017;3(Suppl 1):157-69. doi: 10.18053/jctres.03.2017S1.002. [PubMed: 28670625]. [PubMed Central: PMC5489132].

2. Alinejad S, Zamani N, Abdollahi M, Mehrpour O. A Narrative Review of Acute Adult Poisoning in Iran. Iran J Med Sci. 2017;42(4):327-46. [PubMed: 28761199]. [PubMed Central: PMC5523040].

3. Nili-Ahmadabadi H, Emami MH, Omidifar N. Effectiveness of Quercus brantii hydroalcoholic extract on dyspepsia: A randomized, double blind clinical trial. J Educ Health Promot. 2017;6:62. doi: 10.4103/jehp.jehp_31_17. [PubMed: 28852653]. [PubMed Central: PMC5561684].

4. Lanzotti V. The analysis of onion and garlic. J Chromatogr A. 2006;1112(1-2):3-22. doi: 10.1016/j.chroma.2005.12.016. [PubMed: 16388813].

5. Jannat K, Rahman T, Rahmatullah M. Traditional uses, phytochemicals and pharmacological properties of Allium tuberosum Rottler ex spreng. J Med Plants Stud. 2019;7:214-20.

6. Rastogi S, Pandey MM, Rawat AK. Traditional herbs: a remedy for cardiovascular disorders. Phytomedicine. 2016;23(11):1082-9. doi: 10.1016/j.phymed.2015.10.012. [PubMed: 26656228].

7. Aslani N, Entezari MH, Askari G, Maghsoudi Z, Maracy MR. Effect of Garlic and Lemon Juice Mixture on Lipid Profile and Some Cardiovascular Risk Factors in People 30-60 Years Old with Moderate Hyperlipidaemia: A Randomized Clinical Trial. Int J Prev Med. 2016;7:95. doi: 10.4103/2008-7802.187248. [PubMed: 27563431]. [PubMed Central: PMC4977979].

8. Wang HP, Yang J, Qin LQ, Yang XJ. Effect of garlic on blood pressure: a meta-analysis. J Clin Hypertens (Greenwich). 2015;17(3):223-31. doi: 10.1111/jch.12473. [PubMed: 25557383].

9. Nili-Ahmadabadi A, Ali-Heidar F, Ranjbar A, Mousavi L, Ahmadimoghaddam D, Larki-Harchegani A, et al. Protective effect of amlodipine on diazinon-induced changes on oxidative/antioxidant balance in rat hippocampus. Res Pharm Sci.2018;13(4):368-76. doi:10.4103/17355362.235164. [PubMed: 30065770]. [PubMed Central: PMC6040164].

10. Hassani S, Maqbool F, Salek-Maghsoudi A, Rahmani S, Shadboorestan A, Nili-Ahmadabadi A, et al. Alteration of hepatocellular antioxidant gene expression pattern and biomarkers of oxidative damage in diazinon-induced acute toxicity in Wistar rat: A time-course mechanistic study. EXCLIJ. 2018;17:57-71. doi: 10.17179/excli2017-760. [PubMed: 29383019]. [PubMed Central: PMC5780620]. 
11. Zeinvand-Lorestani H, Nili-Ahmadabadi A, Balak F, Hasanzadeh G, Sabzevari O. Protective role of thymoquinone against paraquatinduced hepatotoxicity in mice. Pestic Biochem Physiol. 2018;148:16-21. doi:10.1016/j.pestbp.2018.03.006. [PubMed: 29891368].

12. Nili-Ahmadabadi A, Alibolandi P, Ranjbar A, Mousavi L, NiliAhmadabadi H, Larki-Harchegani A, et al. Thymoquinone attenuates hepatotoxicity and oxidative damage caused by diazinon: an in vivo study. Res Pharm Sci. 2018;13(6):500-8. doi: 10.4103/1735-5362.245962. [PubMed: 30607148]. [PubMed Central: PMC6288994].

13. Kalantari H, Danesh Pajou M, Kheradmand P, Goodarzian M, Zeidooni L. Nephroprotective Effect of Hydroalcoholic Extract Allium jesdianum Boiss against Carbon Tetrachloride Induced Nephrotoxicity via Stress Oxidative in Mice. Pharm Sci. 2018;24(2):89-96. doi: 10.15171/ps.2018.14.

14. Pollock G, Minuk GY. Diagnostic considerations for cholestatic liver disease.J Gastroenterol Hepatol. 2017;32(7):1303-9. doi: 10.111//jgh.13738. [PubMed: 28106928].

15. Ozougwu JC, Obiukwu CE, Obimba KC, Akortha EE, Usanga VU, Elom MO. Comparative restorative effects of plant extracts against acetaminophen-induced liver toxicity. J Chem Pharm Res. 2016;8(3):503-11.

16. Tenkanidiyoor YS, Vasudeva V, Rao S, Gowda D, Rao C, Sanjeev G, et al. Haematopoietic, Antioxidant and Membrane Stabilizing Property of Diallyl Disulphide in Irradiated Mice. J Clin Diagn Res. 2016;10(2):BF015. doi: 10.7860/JCDR/2016/16371.7246. [PubMed: 27042448]. [PubMed Central: PMC4800513].

17. Noh JR, Kim YH, Hwang JH, Choi DH, Kim KS, Oh WK, et al. Sulforaphane protects against acetaminophen-induced hepatotoxicity. Food Chem Toxicol. 2015;80:193-200. doi: 10.1016/j.fct.2015.03.020. [PubMed: 25818464].

18. Honmore V, Kandhare A, Zanwar AA, Rojatkar S, Bodhankar S, Natu A. Artemisia pallens alleviates acetaminophen induced toxicity via modulation of endogenous biomarkers. Pharm Biol. 2015;53(4):571-81. doi: 10.3109/13880209.2014.934382. [PubMed: 25339313].

19. Wang X, Wu Q, Liu A, Anadon A, Rodriguez JL, Martinez-Larranaga MR, et al. Paracetamol: overdose-induced oxidative stress toxicity, metabolism, and protective effects of various compounds in vivo and in vitro. Drug Metab Rev. 2017;49(4):395-437. doi: 10.1080/03602532.2017.1354014. [PubMed: 28766385].

20. Kandemir FM, Kucukler S, Eldutar E, Caglayan C, Gulcin I. Chrysin Protects Rat Kidney from Paracetamol-Induced Oxidative Stress, Inflammation, Apoptosis, and Autophagy: A Multi-Biomarker Approach. Sci Pharm. 2017;85(1). doi: 10.3390/scipharm85010004. [PubMed: 28134775]. [PubMed Central: PMC5388142].

21. Amin KA, Hashem KS, Alshehri FS, Awad ST, Hassan MS. Antioxidant and Hepatoprotective Efficiency of Selenium Nanoparticles Against Acetaminophen-Induced Hepatic Damage. Biol Trace Elem Res. 2017;175(1):136-45. doi: 10.1007/s12011-016-0748-6. [PubMed: 27220627].

22. Singh MP, Kim KY, Kim HY. Methionine sulfoxide reductase A deficiency exacerbates acute liver injury induced by acetaminophen. Biochem Biophys Res Commun. 2017;484(1):189-94. doi: 10.1016/j.bbrc.2017.01.025. [PubMed: 28104395].

23. Xie W, Jiang Z, Wang J, Zhang X, Melzig MF. Protective effect of hyperoside against acetaminophen (APAP) induced liver injury through enhancement of APAP clearance. Chem Biol Interact. 2016;246:11-9. doi: 10.1016/j.cbi.2016.01.004. [PubMed: 26772156].

24. Gonzalez LT, Minsky NW, Espinosa LE, Aranda RS, Meseguer JP, Perez PC. In vitro assessment of hepatoprotective agents against damage induced by acetaminophen and CCl4. BMC Complement Altern
Med. 2017;17(1):39. doi: 10.1186/s12906-016-1506-1. [PubMed: 28086854]. [PubMed Central: PMC5234107].

25. Sciuto AM. Antioxidant Properties of Glutathione and its Role in Tissue Protection. Oxidants, Antioxidants, and Free Radicals. 2017. p.171-91. doi: 10.1201/9780203744673-9.

26. Ilyas S, Rehman A. Oxidative stress, glutathione level and antioxidant response to heavy metals in multi-resistant pathogen, Candida tropicalis. Environ Monit Assess. 2015;187(1):4115. doi: 10.1007/s10661-0144115-9. [PubMed: 25384372].

27. Singh MP, Kwak GH, Kim KY, Kim HY. Methionine sulfoxide reductase A protects hepatocytes against acetaminophen-induced toxicity via regulation of thioredoxin reductase 1 expression. Biochem Biophys Res Commun. 2017;487(3):695-701. doi: 10.1016/j.bbrc.2017.04.119. [PubMed: 28442342].

28. Du K, Ramachandran A, Jaeschke H. Oxidative stress during acetaminophen hepatotoxicity: Sources, pathophysiological role and therapeutic potential. Redox Biol. 2016;10:148-56. doi: 10.1016/j.redox.2016.10.001. [PubMed: 27744120]. [PubMed Central: PMC5065645].

29. Akinyemi AJ, Lekan Faboya AP, Awonegan IO, Anadozie S, Oluwasola TA. Antioxidant and anti-Acetylcholinesterase activities of essential oils from garlic (Allium sativum) Bulbs. Int J Plant Res. 2018;31.

30. Puri SK, Habbu PV, Kulkarni PV, Kulkarni VH. Hepatoprotective activity of fungal endophytic fractions of andrographis paniculata (burm. f.) wall nees. leaves in paracetamol and ethanol induced hepatotoxicity. Int J Pharm Sci Res. 2019;10(1). doi:10.13040/ijpsr.0975-8232.10(1).97107.

31. Niki E. Lipid peroxidation: physiological levels and dual biological effects. Free Radic Biol Med. 2009;47(5):469-84. doi: 10.1016/j.freeradbiomed.2009.05.032. [PubMed: 19500666].

32. Sharma S, Kota K, Ragavendhra P. HRBC Membrane Stabilization as a study tool to explore the Anti Inflammatory activity of Alliumcepa Linn.-Relevance for 3R. J Adv Med Dent Scie Res. 2018;6(6):30-4. doi: $10.21276 /$ jamdsr.

33. Sakthivel AP. Microwave Assisted Synthesis and Characterization of Silver Nanoparticles using Tridax procumbens and its AntiInflammatory Activity against Human Blood Cells. J Nanomater Mol Nanotechnol. 2015;4(1). doi: 10.4172/2324-8777.1000170.

34. Iwakiri Y, Kim MY. Nitric oxide in liver diseases. Trends Pharmacol Sci. 2015;36(8):524-36. doi:10.1016/j.tips.2015.05.001. [PubMed: 26027855]. [PubMed Central: PMC4532625].

35. Moles A, Torres S, Baulies A, Garcia-Ruiz C, Fernandez-Checa JC. Mitochondrial-Lysosomal Axis in Acetaminophen Hepatotoxicity. Front Pharmacol. 2018;9:453. doi: 10.3389/fphar.2018.00453. [PubMed: 29867464]. [PubMed Central: PMC5968389].

36. Xie W, Wang M, Chen C, Zhang X, Melzig MF. Hepatoprotective effect of isoquercitrin against acetaminophen-induced liver injury. Life Sci. 2016;152:180-9. doi:10.1016/j.lfs.2016.04.002. [PubMed: 27049115].

37. Hinson JA, Roberts DW, James LP. Mechanisms of acetaminopheninduced liver necrosis. Handb Exp Pharmacol. 2010;(196):369-405. doi: 10.1007/978-3-642-00663-0_12. [PubMed: 20020268]. [PubMed Central: PMC2836803].

38. Banerjee SK, Mukherjee PK, Maulik SK. Garlic as an antioxidant: the good, the bad and the ugly. Phytother Res. 2003;17(2):97-106. doi: 10.1002/ptr.1281. [PubMed: 12601669].

39. Suman S, Shukla Y. Diallyl Sulfide and Its Role in Chronic Diseases Prevention. Adv Exp Med Biol. 2016;929:127-44. doi: 10.1007/978-3-31941342-6_6. [PubMed: 27771923]. 